\title{
Effects of Nanostructured Back Reflectors on the External Quantum Efficiency in Thin Film Solar Cells
}

\author{
Chingmei Hsu', George F. Burkhard², Michael D. McGehee ${ }^{1}$, and Yi Cui ${ }^{1}(\bowtie)$ \\ ${ }^{1}$ Department of Materials Science and Engineering, Stanford University, Stanford, California 94305, USA \\ ${ }^{2}$ Department of Applied Physics, Stanford University, Stanford, California 94305, USA \\ Received: 5 October 2010 / Revised: 21 October 2010 / Accepted: 24 October 2010 \\ C The Author(s) 2010. This article is published with open access at Springerlink.com
}

\begin{abstract}
Hydrogenated amorphous $\mathrm{Si}(\alpha-\mathrm{Si}: \mathrm{H})$ is a promising material for photovoltaic applications due to its low cost, high abundance, long lifetime, and non-toxicity. We demonstrate a device designed to investigate the effect of nanostructured back reflectors on quantum efficiency in photovoltaic devices. We adopt a superstrate configuration so that we may use conventional industrial light trapping strategies for thin film solar cells as a reference for comparison. We controlled the nanostructure parameters via a wafer-scale self-assembly technique and systematically studied the relation between nanostructure size and photocurrent generation. The gain/loss transition at short wavelengths showed red-shifts with decreasing nanostructure scale. In the infrared region the nanostructured back reflector shows large photocurrent enhancement with a modified feature scale. This device geometry is a useful archetype for investigating absorption enhancement by nanostructures.
\end{abstract}

\section{KEYWORDS}

Solar cells, back reflector, scattering, solar energy, amorphous silicon

\section{Introduction}

Solar technology is a leading candidate for clean energy production. Silicon is an ideal material for photovoltaic (PV) applications due to its low toxicity, high abundance, long term stability, and welldeveloped processing technologies. Crystalline Si solar cells currently dominate the photovoltaic market despite requiring more material and more intense manufacturing processes than their thin film counterparts. Thin film technologies such as those based on cadmium telluride (CdTe), copper indium gallium diselenide (CIGS), and hydrogenated amorphous silicon $(\alpha-\mathrm{Si}: \mathrm{H})$ require less material with active layer thicknesses of approximately 1-2 $\mu \mathrm{m}$. Although $\alpha-\mathrm{Si}: \mathrm{H}$ provides the advantage of lower material costs than crystalline silicon, the thin layers do not absorb light effectively, resulting in poor cell performance. Because the material is amorphous there are many defects, which result in a small minority carrier diffusion length. If a film of active material with a thickness approximately equal to the minority carrier diffusion length could be made to absorb all of the light, the open-circuit voltage $\left(V_{\text {oc }}\right)$, short-circuit current $\left(J_{\text {sc }}\right)$, and fill factor (FF) of the device would be greater than that of a thicker cell.

Many approaches have been proposed to reduce the mismatch between ideal electrical and optical thicknesses, including anti-reflection coatings (ARCs) and surface textures on the fronts or backs of the cells.

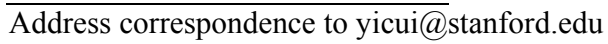


ARCs do not provide transmittance over the entire solar spectrum. Conventional texturing with feature sizes of $2-10 \mu \mathrm{m}$ for crystalline Si cells is not suitable for thin film cells with thicknesses in the micron range [1-3]. Several strategies have been proposed to address these issues, such as incorporating random nano-scale surface texturing [4-6] and utilizing plasmon resonance to enhance absorption [7-10].

Several studies have demonstrated highly absorptive periodic nanostructured solar cells [11-15]. A different light-trapping strategy, which may not cause recombination losses in thin film solar cells, is to incorporate metal nanoparticles into the active layer to enhance light scattering, effectively lengthening the optical path length [7-9]. Localized surface plasmons are collective oscillations of the conduction electrons in metal nanoparticles. Movement of the conduction electrons upon excitation with incident light results in a buildup of oscillating dipoles on the particle surface, allowing for resonance at specific wavelengths. For off-resonant wavelengths, absorption is suppressed leading to enhancement in the scattering cross-section. In a thin film, this scattering enhancement can result in an increase in absorption in the surrounding material Use of plasmon resonance has led to photocurrent enhancement at specific wavelengths and overall device power efficiencies of $8 \%, 8.3 \%$, and $40 \%$ in GaAs [16], $\alpha$-Si [17], and organic photovoltaics [18] respectively. Earlier work has demonstrated light scattering from metal particles of various shapes, sizes, materials, and dielectric environments, and suggested design rules for particle-enhanced solar cells [19]. Most strategies have focused on placing metal particles on the front of the cells, which can lead to strong absorption losses near the resonant frequencies of the particles. A variety of device geometries have been proposed to address this issue, such as locating nanoparticles at the rear of the device or depositing the active layer on a patterned back reflector [11, 12, 20, 21]. These strategies eliminate the transmittance losses associated with having scatterers at the front of the cells while still promoting scattering events. However, the experimental influence of a periodically patterned back reflector is still unclear.

In this work, we chose the superstrate configuration so that conventional industry standards could be used as controls. We compared our devices to commercially available devices, which use textured fluorine doped tin oxide (FTO) as both an ARC and an electrode. We performed absorption and external quantum efficiency (EQE) measurements to confirm that absorption enhancement resulted in photocurrent enhancement. By synthesizing and patterning uniform nanoparticles, we also investigated the dependence of absorption on the shape, size, and feature-density of the nanostructured back reflectors.

\section{Experimental}

Figure 1 illustrates the fabrication process and a typical geometry used in these studies. $310 \mathrm{~nm} \alpha$-Si:H $p-i-n$ structures $(p-i-n 10 \mathrm{~nm}-280 \mathrm{~nm}-20 \mathrm{~nm})$ were fabricated by plasma enhanced chemical vapor deposition on FTO glass. A $10 \mathrm{~nm}$ layer of indium tin oxide (ITO) was sputtered by radio frequency (RF) sputtering with a $5 \mathrm{~mm} \times 5 \mathrm{~mm}$ device area defined by a metal shadow mask. Controlled sizes of silicon dioxide particles were deposited via the LangmuirBlodgett method, as described in our previous work [14]. In order to study the influence of particle parameters on the optical properties, several different silica particles were employed in this study. Reactive ion etching with a mixture of sulfur hexafluoride $\left(\mathrm{SF}_{6}\right)$ and chlorodifluoromethane $\left(\mathrm{CHClF}_{2}\right)$ was performed to in order to control the particle shapes and density, and the spacing between particles.

Because of the etching process, particles changed from spheres to hemispheres to disks, as shown in Figs. 2(a) and 2(d). $300 \mathrm{~nm} \mathrm{Ag} \mathrm{was} \mathrm{deposited} \mathrm{by} \mathrm{direct}$ current (DC) sputtering, which acted as both a back reflector and a back contact. Figs. 2(b) and 2(c) show top views of the devices after Ag metallization, with the corresponding silica features in Figs. 2(e) and 2(f).

\section{Results and discussion}

We investigated the effects of six different nanostructures on the quantum efficiencies of $\alpha$-Si cells. Figures of merit for all devices are shown in Table 1. The nanostructures used included medium spherical nanostructures $\left(\mathrm{M}_{\mathrm{s}}\right)$, medium hemispherical nanostructures $\left(M_{h}\right)$, medium disk nanostructures $\left(M_{d}\right)$, 


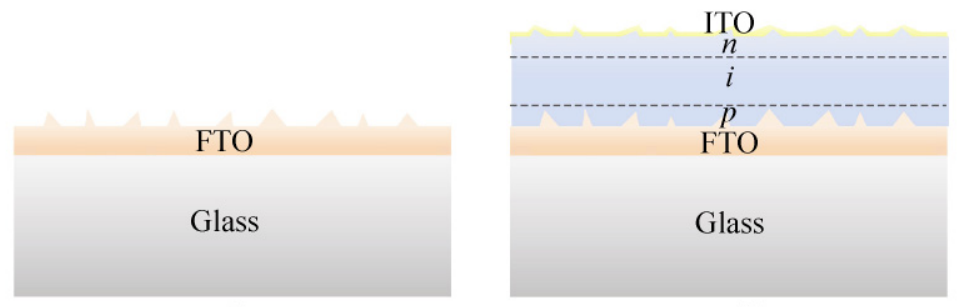

(a)

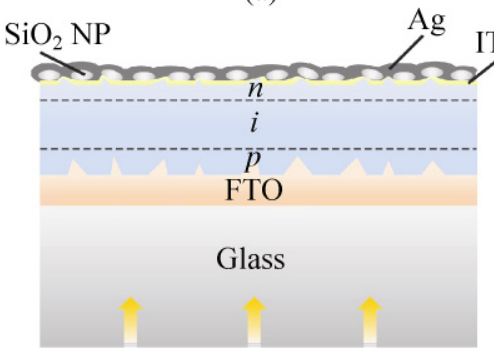

(d)

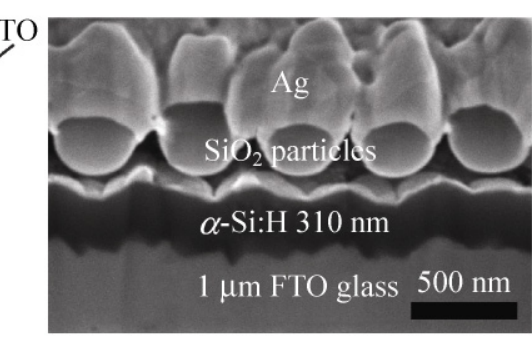

(e)

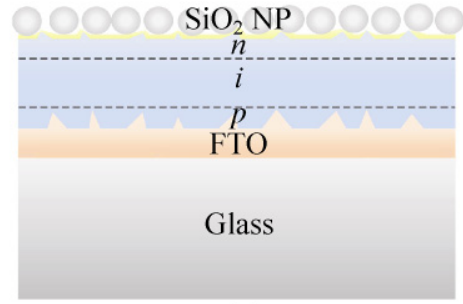

(c)

Figure 1 Fabrication process of nanostructured thin film solar cells. (a, b) $310 \mathrm{~nm} \alpha$-Si:H $p-i-n$ structures $(p-i-n 10 \mathrm{~nm}-280 \mathrm{~nm}-20 \mathrm{~nm})$ and a $10 \mathrm{~nm}$ layer of indium tin oxide (ITO) were deposited on fluorine doped tin oxide (FTO) glass in sequence. (c) A monolayer of silicon dioxide particles was deposited by the Langmuir-Blodgett method and (d) reactive ion etching was performed to reduce particle dimensions and followed by deposition of a layer of $300 \mathrm{~nm} \mathrm{Ag}$. (e) Scanning electron microscope (SEM) cross section of the as-made device, $\mathrm{M}_{\mathrm{s}}$

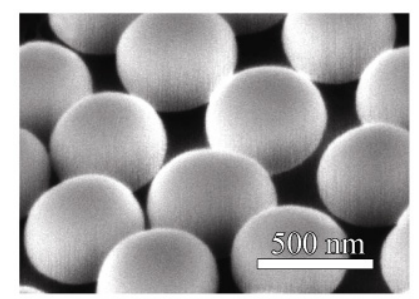

(a)

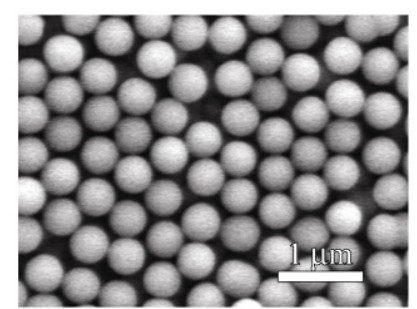

(b)

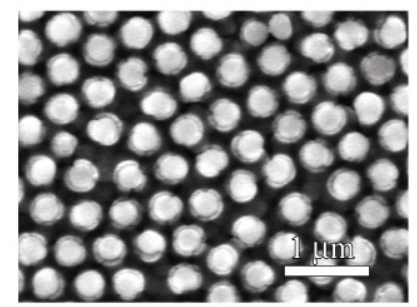

(c)

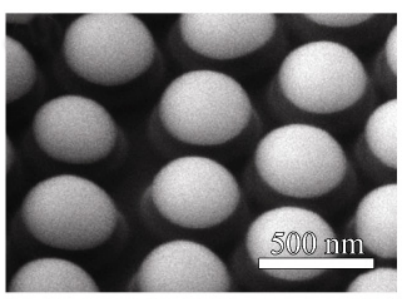

(d)

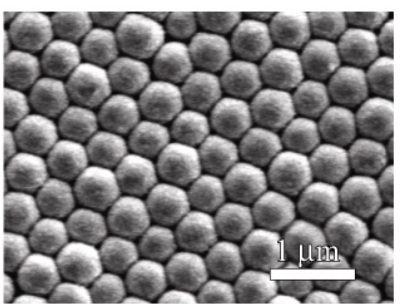

(e)

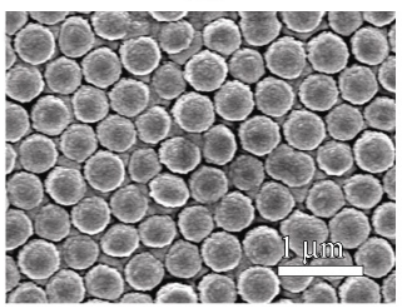

(f)

Figure 2 SEM images of (a) spherical $\mathrm{SiO}_{2}$ nanoparticles (d) hemispherical $\mathrm{SiO}_{2}$ nanoparticles, $45^{\circ}$ tilted. Top view of (b) spherical $\mathrm{SiO}_{2}$ nanoparticles (c) disk $\mathrm{SiO}_{2}$ nanoparticles. (e, f) after metallization corresponding to (b) and (c)

Table 1 Details of the device parameters and wavelengths of EQE gain/loss transitions

\begin{tabular}{ccccc}
\hline & Shape & $\begin{array}{c}\text { Periodicity } \\
(\mathrm{nm})\end{array}$ & $\begin{array}{c}\text { Silica size } \\
(\mathrm{nm})\end{array}$ & $\begin{array}{c}\text { EQE transition } \\
(\mathrm{nm})\end{array}$ \\
\hline $\mathrm{M}_{\mathrm{s}}$ & Sphere & 515 & 437 & 525 \\
$\mathrm{M}_{\mathrm{h}}$ & Hemisphere & 515 & 225 & 545 \\
$\mathrm{M}_{\mathrm{d}}$ & Disk & 515 & 161 & 570 \\
$\mathrm{~S}_{\mathrm{s}}$ & Sphere & 327 & 268 & 530 \\
$\mathrm{~S}_{\mathrm{h}}$ & Hemisphere & 327 & 268 & 555 \\
$\mathrm{~S}_{\mathrm{d}}$ & Disk & 327 & 226 & 560 \\
\hline
\end{tabular}

small spherical nanostructures $\left(S_{\mathrm{s}}\right)$, small hemispherical nanostructures $\left(\mathrm{S}_{\mathrm{h}}\right)$, and small disk nanostructures $\left(\mathrm{S}_{\mathrm{d}}\right)$. $\mathrm{M}_{\mathrm{s}-\mathrm{h}}$ refers to the transition state between medium spherical and hemispherical nanostructures. The external quantum efficiencies and absorption spectra of cells made with nanostructures of various geometries and a constant periodicity of $550 \mathrm{~nm}$ are shown in Figs. 3 and 4(a). While the nanostructured cells showed enhanced photocurrent response compared to the reference cell at shorter wavelengths, all of the cells had decreased photocurrent responses at wavelengths longer than approximately $550 \mathrm{~nm}$. The quantum efficiency spectra could be tuned by tweaking the

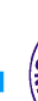

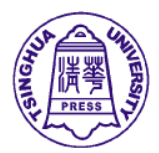

Springer 


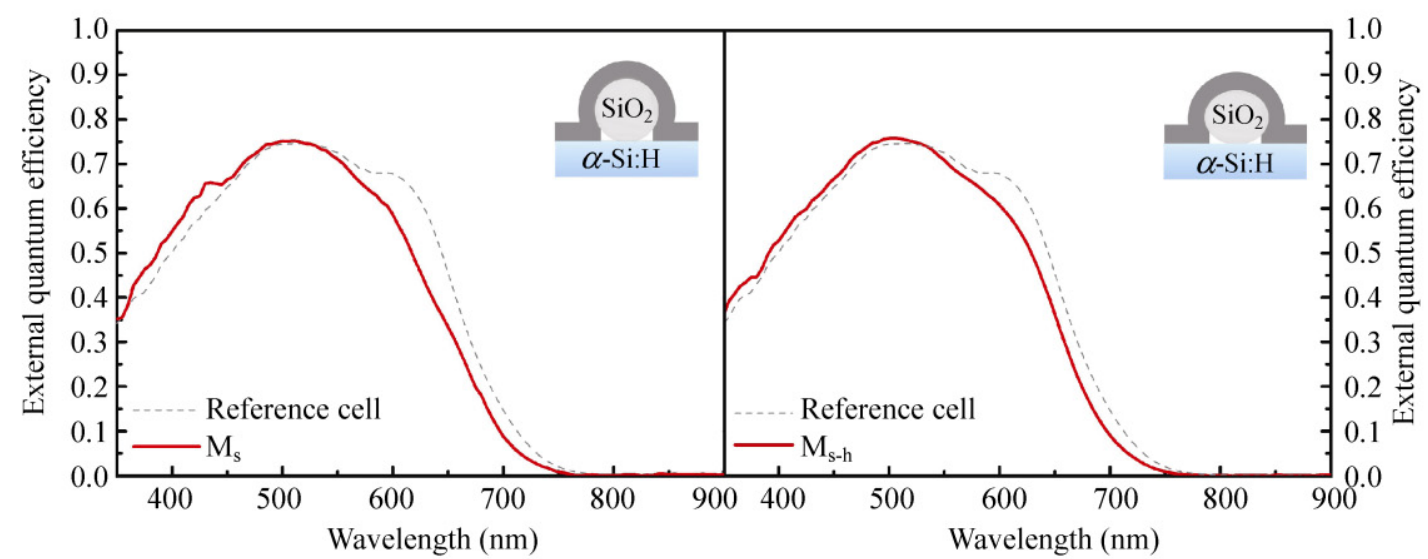

(a)

(b)

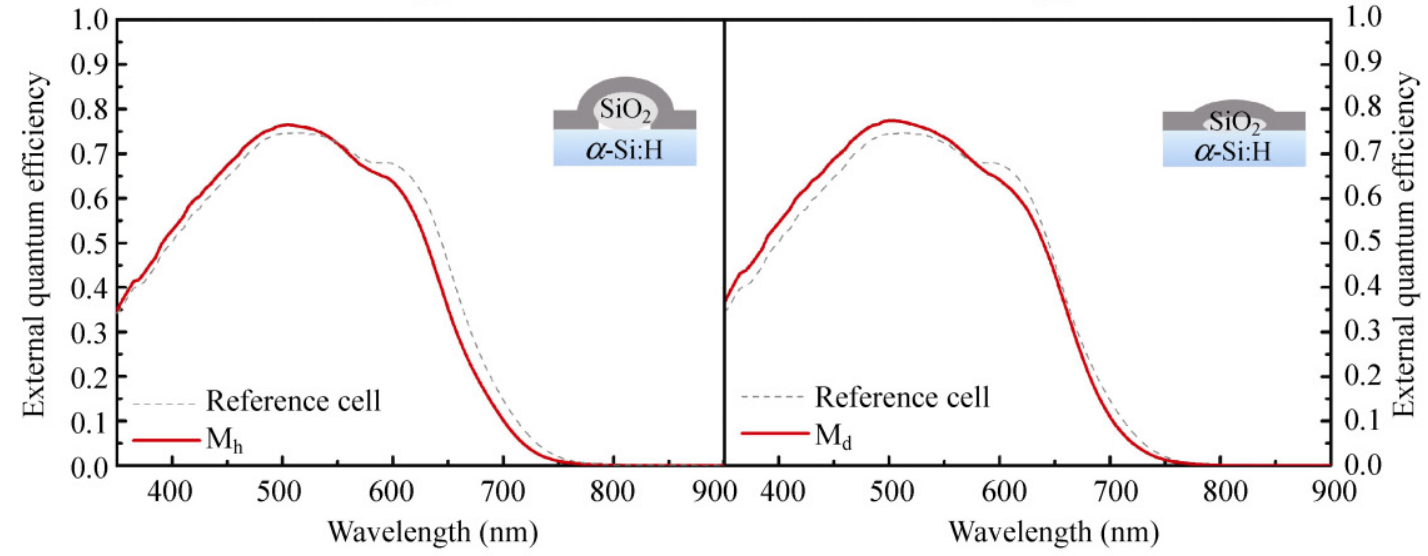

(c)

(d)

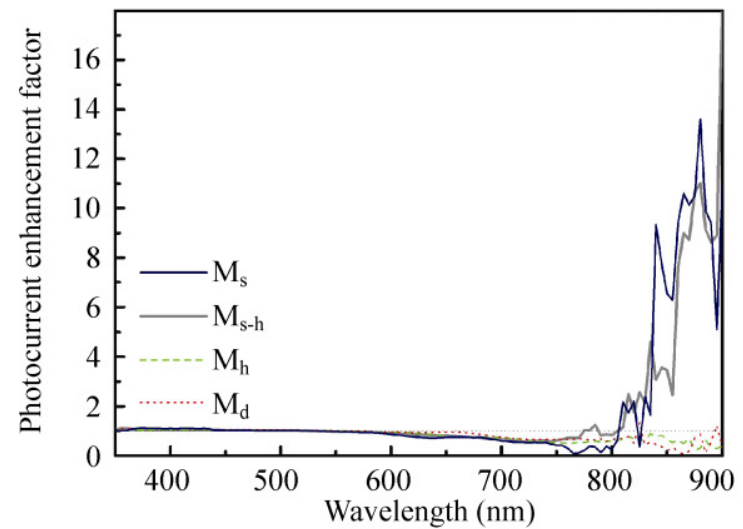

(e)

Figure 3 External quantum efficiencies of devices (a) $M_{s}$, (b) $M_{s-h}$, (c) $M_{h}$, and (d) $M_{d}$, and (e) EQEs of M-series devices normalized by the EQE of the reference cell. The inserted illustrations show the interfaces between silicon dioxide particles, $\alpha-\mathrm{Si}: \mathrm{H}$, and $\mathrm{Ag}$

parameters of the nanostructure. For instance, the photocurrent gain/loss transitions occurred at $525 \mathrm{~nm}$, $530 \mathrm{~nm}, 545 \mathrm{~nm}$, and $570 \mathrm{~nm}$ for $\mathrm{M}_{\mathrm{s}}, \mathrm{M}_{\mathrm{s}-\mathrm{h}}, \mathrm{M}_{\mathrm{h}}$, and $\mathrm{M}_{\mathrm{d}}$ respectively. Photocurrent enhancements before the gain/loss transition at shorter wavelengths were $3.15 \%$, $3.50 \%, 4.21 \%$, and $4.96 \%$. Interestingly, the shortwavelength gain/loss transitions showed red-shifts with increasing ratio of annular cross-sectional area of $\mathrm{Ag}$ to total cross-sectional area of $\mathrm{SiO}_{2}$, with the same periodicity, for both the medium (M) and small (S) series. The same trend was also observed in the absorption spectra. This result indicates that nanostructures enhance scattering at shorter wavelengths. The effects of nanostructure shape on quantum efficiency are 
less clear since the etching process changes not only the shapes but also the diameters of nanostructures. Though both $S_{s}$ and $S_{h}$ had similar dimensions, they showed red-shifts, at $530 \mathrm{~nm}$ and $555 \mathrm{~nm}$ respectively. $\mathrm{S}_{\mathrm{h}}$ showed an $82 \%$ increase in photocurrent over $\mathrm{S}_{\mathrm{s}}$ in this range, indicating that hemispherical dielectric nanomaterials scatter more efficiently than spherical ones at shorter wavelengths. Though strong absorption enhancement was obtained, particularly at long wavelengths, reduction of photocurrent generation occurred at approximately $530 \mathrm{~nm}$ in each case. This suggests that the additional absorption observed at this wavelength may be due to absorption in small $\mathrm{Ag}$ islands, or is occurring very close to the Si surface and not in the active region of the cell. Additionally, it is possible that incident light is wave guided through total internal reflection at the interfaces in $\alpha$-Si/air $/ \mathrm{SiO}_{2}$ and eventually is absorbed by surrounding $\mathrm{Ag}$ islands. After performing etching to tune particle size and shapes, absorption losses from the patterned back reflector were dramatically reduced. The materials loss characterization can be found in Fig. 4(b). To investigate the photocurrent response at long wavelengths, the external quantum efficiencies of the nanoparticle-patterned cells were normalized by that of the reference cell, as shown in Fig. 3(e). Photocurrent enhancement in the active layer at long wavelengths can be primarily ascribed to light trapping provided by the nanostructured back reflector scattering incident light back into the substrate. As Fig. 4(b) indicates, in addition to enhancing absorption

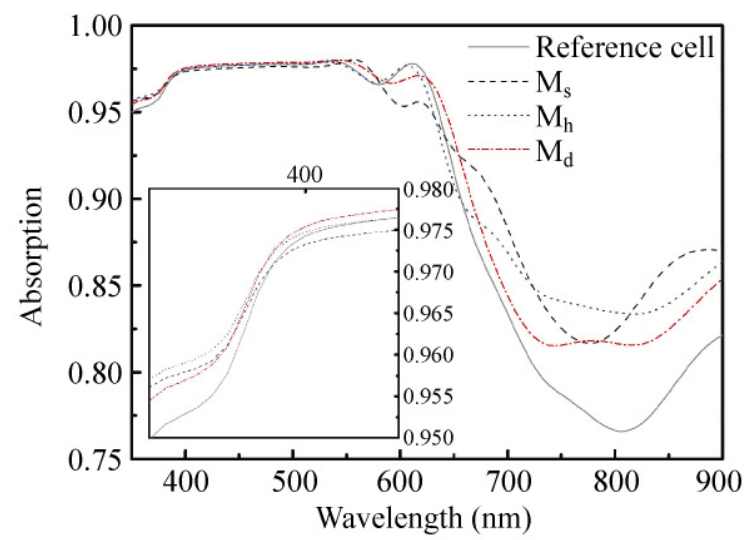

(a) in the active layer of the cell, the nanostructure also enhances parasitic absorption in the silver electrode. At wavelengths between $810 \mathrm{~nm}$ and $900 \mathrm{~nm} \mathrm{M}_{\mathrm{s}}$ and $M_{s-h}$ show 14-fold enhancement, while others show reduction of photocurrent generation, even at long wavelengths. This reduction may be due to damage to the $\alpha$-Si during the long etching process. Because etching can damage $\alpha-\mathrm{Si}$, there is a tradeoff between creating the optimal nanostructure shape for efficient scattering and preserving the electrical properties of the cell.

\section{Conclusion}

We have shown that by incorporating metal nanostructured back reflectors, we can tune the localized surface plasmon enhanced absorption at both shorter and longer wavelengths by varying the shapes, feature sizes, and periodicities of the nanostructures. The cell design is not optimal for overall photocurrent enhancement; the nanostructured back reflector itself exhibits significant absorption loss across the whole spectrum, absorbing $30 \%$ of incident light even at long wavelength, as shown in Fig. 4(b). EQE is enhanced and suppressed at short and long wavelengths respectively. For short wavelengths, EQE is improved, since the scattering of the nanostructured silver substrate helps to lengthen the optical path, enhancing absorption. EQE suppression at long wavelengths may be due to the fact that - at these wavelengths-absorption in the nanostructured back reflector is much stronger than absorption in the $\alpha$-Si. Scattering centers, even at

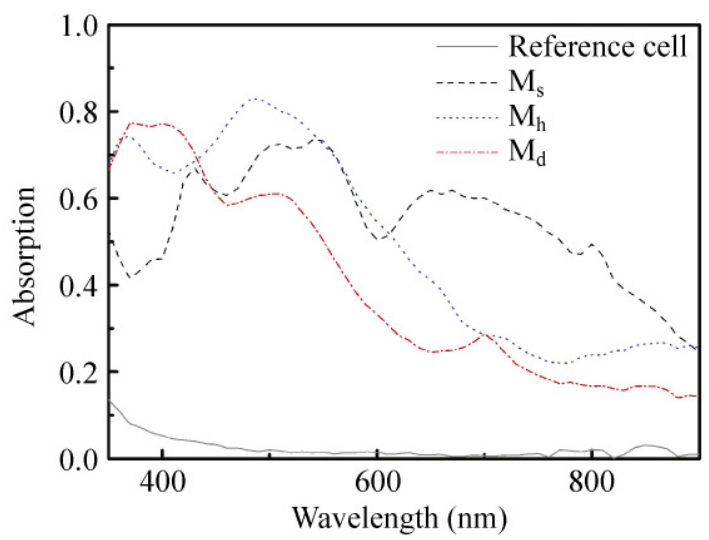

(b)

Figure 4 (a) The absorption spectra of M-series devices. (b) The absorption spectra of nanostructured back reflectors without absorber layers (absorption loss) 
the back of the substrate, may not always improve the efficiency, particularly in the case that the scatterers are very lossy. However, this work serves as a guide to improve device geometry for absorption over the entire spectrum via enhanced scattering.

\section{Acknowledgements}

We thank Dr. Tiemin Zhao and Dr. Zongfu Yu for material deposition and optical discussion.

Open Access: This article is distributed under the terms of the Creative Commons Attribution Noncommercial License which permits any noncommercial use, distribution, and reproduction in any medium, provided the original author(s) and source are credited.

\section{References}

[1] Green, M. A. Lambertian light trapping in textured solar cells and light-emitting diodes: Analytical solutions. Prog. Photovoltaics 2002, 10, 235-241.

[2] Yablonovitch, E. Statistical ray optics. J. Opt. Soc. Am. 1982, 72, 899-907.

[3] Campbell, P.; Green, M. A. Light trapping properties of pyramidally textured surfaces. J. Appl. Phys. 1987, 62, 243-249.

[4] Fahr, S.; Rockstuhl, C.; Lederer, F. Engineering the randomness for enhanced absorption in solar cells. Appl. Phys. Lett. 2008, 92, 171114.

[5] Franken, R. H.; Stolk, R. L.; Li, H.; van der Werf, C. H. M.; Rath, J. K.; Schropp, R. E. I. Understanding light trapping by light-scattering textured back electrodes in thin-film $n-i-p$ silicon solar cells. J. Appl. Phys. 2007, 102, 014503.

[6] Müller, J.; Rech, B.; Springer, J.; Vanecek, M. TCO and light trapping in silicon thin film solar cells. Sol. Energy 2004, 77, 917-930.

[7] Stuart, H. R.; Hall, D. G. Absorption enhancement in silicon-on-insulator waveguides using metal island films. Appl. Phys. Lett. 1996, 69, 2327-2329.

[8] Stuart, H. R.; Hall, D. G. Island size effects in nanoparticleenhanced photodetectors. Appl. Phys. Lett. 1998, 73, 38153817.

[9] Schaadt, D. M.; Feng, B.; Yu, E. T. Enhanced semiconductor optical absorption via surface plasmon excitation in metal nanoparticles. Appl. Phys. Lett. 2005, 86, 063106.

[10] Pillai, S.; Catchople, K. R.; Trupke, T.; Green, M. A. Surface plasmon enhanced silicon solar cells. J. Appl. Phys. 2007, 101, 093105.

[11] Ferry, V. E.; Verschuuren, M. A.; Li, H. B. T.; Schropp, R. E. I.; Atwater, H. A.; Polman, A. Improved red-response in thin film $\alpha$-Si:H solar cells with soft-imprinted plasmonic back reflectors. Appl. Phys. Lett. 2009, 95, 183503.

[12] Ferry, V. E.; Sweatlock, L. A.; Pacifici, D.; Atwater, H. A. Plasmonic nanostructure design for efficient light coupling into solar cells. Nano Lett. 2008, 8, 4391-4397.

[13] Zhu, J.; Hsu, C. M.; Yu, Z.; Fan, S.; Cui, Y. Nanodome solar cells with efficient light management and self-cleaning. Nano Lett. 2010, 10, 1979-1984.

[14] Hsu, C. M.; Connor, S. T.; Tang, M. X.; Cui, Y. Wafer-scale silicon nanopillars and nanocones by Langmuir-Blodgett assembly and etching. Appl. Phys. Lett. 2008, 93, 133109.

[15] Ferry, V. E.; Verschuuren, M. A.; Li, H. B. T.; Verhagen, E.; Walters, R. J.; Schropp, R. E. I.; Atwater, H. A.; Polman, A. Light trapping in ultrathin plasmonic solar cells. Opt. Express 2010, 18, A237-A245.

[16] Keisuke, N.; Katsuaki, T.; Atwater, H. A. Plasmonic nanoparticle enhanced light absorption in GaAs solar cells. Appl. Phys. Lett. 2008, 93, 121904.

[17] Derkacs, D.; Lim, S. H.; Matheu, P.; Mar, W. ; Yu, E. T. Improved performance of amorphous silicon solar cells via scattering from surface plasmon polaritons in nearby metallic nanoparticles. Appl. Phys. Lett. 2006, 89, 093103.

[18] Morfa, A. J.; Rowlen, K. L.; Reilly, T. H.; Romero, M. J.; van de Lagemaat, J. Plasmon-enhanced solar energy conversion in organic bulk heterojunction photovoltaics. Appl. Phys. Lett. 2008, 92, 013504.

[19] Catchpole, K. R.; Polman, A. Design principles for particle plasmon enhanced solar cells. Appl. Phys. Lett. 2008, 93, 191113.

[20] Beck, F. J.; Polman, A.; Catchpole, K. R. Tunable light trapping for solar cells using localized surface plasmons. $J$. Appl. Phys. 2009, 105, 114310.

[21] Beck, F. J.; Mokkapati, S.; Polman A.; Catchpole, K. R. Asymmetry in light-trapping by plasmonic nanoparticle arrays located on the front or on the rear of solar cells. Appl. Phys. Lett. 2010, 96, 033113. 